\title{
Micromeria rodriguezii (Lamiaceae) en la flora peninsular ibérica
}

\author{
R. SENAR ${ }^{1 *}$, P. P. FERRER-GALLEGO ${ }^{2 *}$, E. LAGUNA² ${ }^{2}$ J. A. ROSSELLÓ³ \& L. SÁEZ ${ }^{4,5}$ \\ ${ }^{1}$ c. César Cataldo, 13, ES-12580 Benicarló, Castellón, España \\ ${ }^{2}$ Servicio de Vida Silvestre, Centro para la Investigación y Experimentación Forestal de la Generalitat Valenciana \\ (CIEF), av. Comarques del País Valencià, 114, ES-46930 Quart de Poblet, Valencia, España \\ ${ }^{3}$ Jardín Botánico-ICBiBE-Unidad Asociada CSIC, Universitat de València, c. Quart, 80, ES-46008 Valencia, España \\ ${ }^{4}$ Sistemàtica i Evolució de Plantes Vasculars, Unitat Associada al CSIC, Botànica, Facultat de Biociències, Universi- \\ tat Autònoma de Barcelona, ES-8193 Bellaterra, Barcelona, España \\ ${ }_{5}^{5}$ Societat d'Història Natural de les Illes Balears, c. Margarida Xirgu, 16, ES-07003 Palma de Mallorca, Islas Baleares, \\ España
}

ORCID iD. R. SENAR: https://orcid.org/0000-0003-3316-897X,

P. P. FERRER-GALLEGO: https://orcid.org/0000-0001-7595-9302, E. LAGUNA: https://orcid.org/0000-0002-9674-2767, J. A. ROSSELLÓ: https://orcid.org/0000-0002-0432-661X, L. SÁEZ: https://orcid.org/0000-0003-4551-2432

Autor para correspondencia: P. P. Ferrer-Gallego (flora.cief@gva.es)

*Ambos autores contribuyen igualmente a este trabajo

Editor: R. Vilatersana

Recibido 26 septiembre 2019; aceptado 21 enero 2020; publicado on line 20 abril 2020

\begin{abstract}
MicRomeria RodRIGUEZI (LAMIACEAE) In THE IBERIAN PENInSULAR FLORA.- Micromeria rodriguezii is an endemic species from the Balearic Islands (Western Mediterranean basin). A population of this species has been found in NE Spain (Castellón province), growing in the margins of a forest track. A study of the plant morphology of this Iberian population is provided. Its conservation status in the Valencian Community is discussed and a management and conservation plan is suggested to be drawn up to ensure its preservation.
\end{abstract}

Key words: Castellón province; chorology; Iberian Peninsula; Lamiaceae; Micromeria.

\section{Resumen}

MicRomerIa RODRIGUEZII (LAMIACEAE) EN LA FLORA PENINSULAR IBÉRICA.- Micromeria rodriguezii es una especie endémica de las Islas Baleares (Mediterráneo occidental). Una población de esta especie se ha encontrado en la provincia de Castellón (España), en el margen de un camino forestal. Se proporciona un estudio de la morfología de las plantas de esta población, así como su estado de conservación en la Comunidad Valenciana. Debido a que esta es la única población ibérica conocida hasta el momento, se debe elaborar un plan de manejo y conservación para garantizar su conservación.

Palabras clave: corología; Lamiaceae; Micromeria; Península Ibérica; provincia de Castellón.

\section{Cómo citar este artículo / Citation}

Senar, R., Ferrer-Gallego, P. P., Laguna, E., Rosselló, J. A. \& Sáez, L. 2020. Micromeria rodriguezii (Lamiaceae) en la flora peninsular ibérica. Collectanea Botanica 39: e006. https://doi.org/10.3989/collectbot.2020.v39.006

\section{Copyright}

(C) 2020 CSIC. This is an open-access article distributed under the terms of the Creative Commons Attribution 4.0 International (CC BY 4.0) License. 


\section{INTRODUCCIÓN}

El género Micromeria Bentham (1829: sub t. 1282) (Lamiaceae) con aproximadamente 70-90 táxones, según autores, es de gran complejidad taxonómica (Puppo et al., 2014, 2015), y está distribuido en las regiones templadas y en las montañas tropicales de todo el mundo, desde las regiones mediterránea y macaronésica hasta el sur de África, India y China (Chater, 1971; Chater \& Guinea, 1972; Pérez de Paz, 1978; Morales, 1991, 1993; Govaerts, 1999; Harley et al., 2004; Bräuchler et al., 2005, 2008; Puppo et al., 2014, 2015; Puppo \& Meimberg, 2015).

Los estudios más analíticos consideran más de nueve táxones de Micromeria dentro de la flora peninsular ibérica e Islas Baleares. Algunos de ellos muestran un área de distribución reducida, pero a su vez con una gran variabilidad morfológica, como por ejemplo el complejo taxonómico M. microphylla- $M$. filiformis. El grupo de táxones relacionados con $M$. microphylla (d'Urv.) Benth., en el sentido más amplio del taxón, se encuentran distribuidos por el Mediterráneo central y occidental [Italia (incl. Sicilia), Malta e Islas Baleares (incl. Mallorca, Menorca, Ibiza y Formentera)] (Greuter et al., 1986; Morales, 1991, 2010; Bolòs \& Vigo, 1996; Gil \& Llorens, 2004).

Los táxones pertenecientes al grupo M. microphylla s. l., son propios de ambientes de roquedos y claros de matorrales, sitios pedregosos de zonas cálidas a baja altitud. Dentro de este grupo, Freyn \& Janka (1874) describieron una nueva especie para la flora de las Islas Baleares a partir de material repartido en el "Iter gallico-hispanicum 1873" de Friedrich Hegelmaier, a la que denominaron M. rodriguezii en honor al botánico Joan Joaquim Rodríguez i Femenías (1839-1905). Este taxón ha sido considerado por algunos autores con nivel subespecífico, bien dentro de M. nervosa (Desf.) Benth., M. filiformis (Aiton) Benth., M. microphylla [o como Satureja microphylla (d'Urv.) Briq.] y Satureja cordata Moris ex Bertol. (véase Fig. 1), o bien como variedad de M. biflora Benth., según las siguientes combinaciones y estatus taxonómico: M. nervosa subsp. rodriguezii (Freyn \& Janka) Nyman, Consp. Fl. Eur.: 590. 1881; M. biflora var. rodriguezii (Freyn \& Janka) Knoche, Fl. Balear.: 346. 1922; M. filiformis subsp. rodriguezii (Freyn \& Janka) Bonafè, Fl. Mallorca 4: 56. 1980; S. cordata subsp. rodriguezii (Freyn \& Janka) O. Bolòs
\& Vigo, Collect. Bot. (Barcelona) 14: 94.1983 (véase también Bolòs et al., 2005: 705); M. microphylla subsp. rodriguezii (Freyn \& Janka) Romo, Fl. Silvestres Baleares: 261. 1994; Satureja microphylla subsp. rodriguezii (Freyn \& Janka) O. Bolòs \& Vigo, Fl. Països Catalans 3: 301. 1996. Estos cambios, así como otras combinaciones nomenclaturales recogidas por Rosselló \& Sáez (2000: 100), reflejan sin duda la complejidad de interpretación y circunscripción de $M$. rodriguezii y la dificultad de una estable ubicación en la sistemática del género.

Ciertos autores han reconocido el valor taxonómico de $M$. rodriguezii, al tiempo que señalaban su variabilidad morfológica, formalizada taxonómicamente en el nivel varietal o con rango de forma, proponiendo lo siguiente: (1) S. rodriguezii var. condensata L. Chodat in Bull. Soc. Bot. Genève, ser. 2, 15: 234.1924 [lectótipo, G00087103, designado por Rosselló \& Sáez (2000: 102) (véase también Bräuchler et al., 2008: 380; Rosselló \& Sáez, 2017: 71): Spain, Balearic Islands, Mallorca, "Torre d'en Pau" III-IV-1921, R. Chodat]; (2) M. rodriguezii f. major Sennen, Treb. Inst. Catalana Hist. Nat. 3: 192.1917 [lectótipo designado por Rosselló \& Sáez, 2000: 101; BC-Sennen 836596 (véase apartado Specimina visa selecta)] (véase Sennen, 1917); (3) M. rodriguezii var. acuminifolia Sennen in Bol. Soc. Ibér. Ci. Nat. 32: 62.1933 (lectótipo designado por Rosselló \& Sáez, 2000: 101; BC-Sennen 836599).

Por otra parte, Mus \& Rosselló (1987) concluyeron que no existían diferencias significativas entre las poblaciones italianas de $M$. microphylla, y las presentes en las Islas Baleares, considerando en consecuencia el nombre $M$. rodriguezii s. $l$. como un sinónimo heterotípico de M. microphylla (Rosselló \& Sáez, 2000: 101-102). Este criterio sintético ha sido seguido también con posterioridad por Rosselló \& Sáez (2000) así como en diferentes revisiones del género en el ámbito ibérico (Morales, 1991, 2010). No obstante, Rivas-Martínez et al. (1992: 217) reivindicaron el valor taxonómico en el rango de especie como Satureja rodriguezii (Freyn \& Janka) Rivas Mart., M. Costa \& Loidi, Itin. Geobot. 6: 217. 1992. De igual modo, tanto Bolòs \& Vigo (1996) como Bräuchler et al. (2008) apoyaron el rango subespecífico para este taxón dentro de $M$. filiformis (véase también The Euro+Med PlantBase; http:// ww2.bgbm.org/EuroPlusMed), al tiempo que Bräuchler et al. (2008) designaron su lectótipo [Spain, Balearic Islands, Ins. Minorca] "Menorca, in valle 


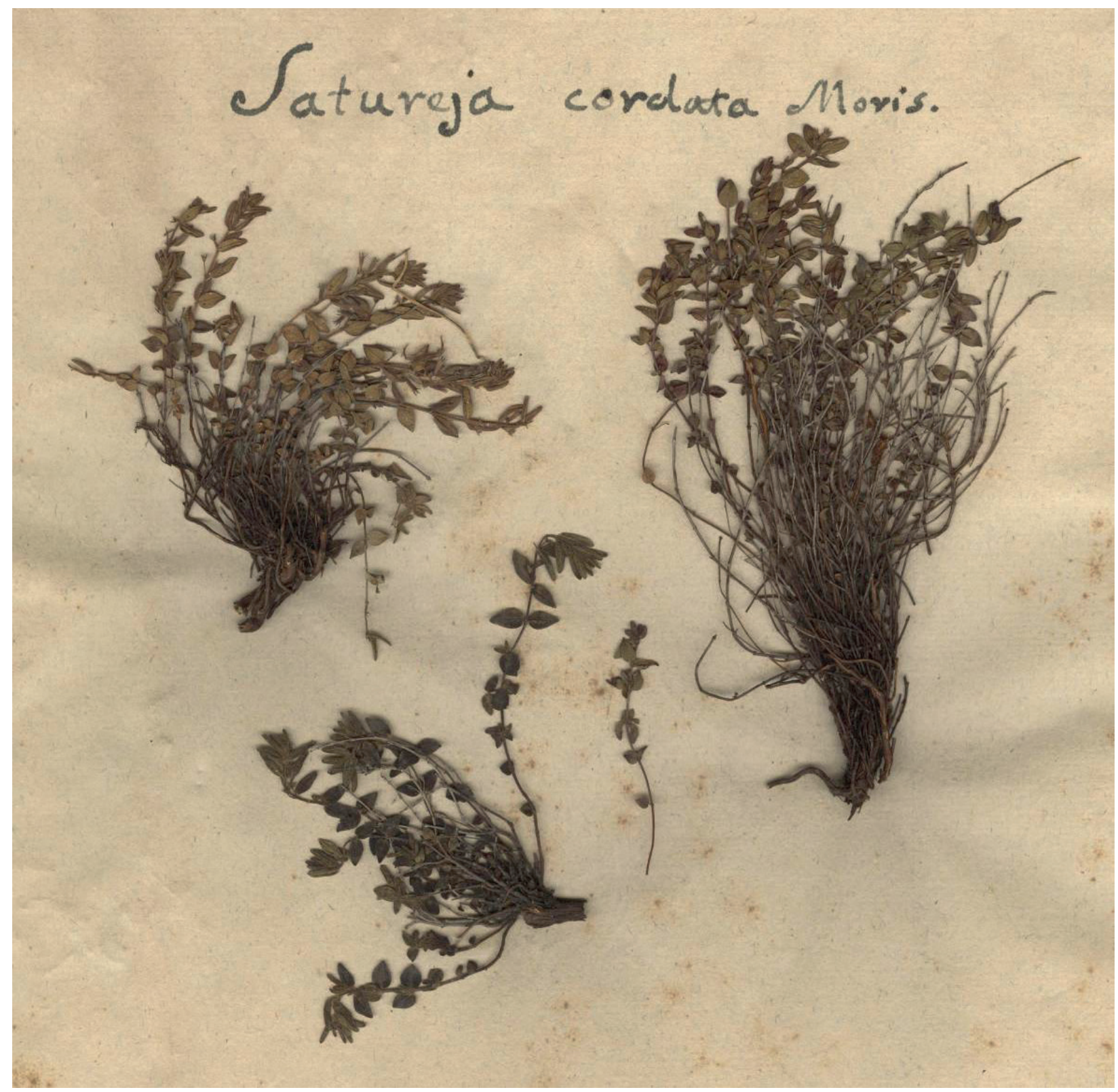

Figura 1. Lectótipo de Satureja cordata, BOLO (s.n.), designado por Bräuchler et al. (2008). Imagen por cortesía de herbario BOLO, reproducida con permiso.

Varranco (sic) del Favaret prope Mahon ad rupes calcareas", 29.3.1873, Hegelmaier, Iter gallico-hispanicum 1873 (STU!', ver Fig. 2; isolectotypo: JE!) (véase también Rosselló \& Sáez, 2017: 71). Entre algunos autores baleáricos, $M$. rodriguezii es reconocida con rango específico o subespecífico (Gil \&

1. El lectótipo se compone de tres hojas vinculadas entre ellas a través de la etiqueta de herbario y el código numérico. Los códigos de barras de los pliegos de herbario que contienen el lectótipo son: STU 1 72456/2011/1 3; STU 1 72456/2011/2 3 y STU 1 72456/2011/3 3. Los tres contienen la misma etiqueta impresa, y el pliego STU 1 $72456 / 2011 / 23$ además la etiqueta original de F. Hegelmaier (Fig. 2).
Llorens, 2004; Fraga-Arguimbau et al., 2004a, b, 2014; Fraga-Arguimbau, 2015).

En este trabajo se comunica la presencia de $M$. rodriguezii en la flora peninsular ibérica, a partir de su hallazgo en la provincia de Castellón. Concretamente, ha sido localizada una población, con dos núcleos muy próximos entre ellos, y con pocos individuos en ambos casos. El estudio tanto de especímenes vivos como de material de herbario nos ha permitido realizar una descripción del material ibérico de esta especie, así como hacer una serie de consideraciones sobre su ecología. 

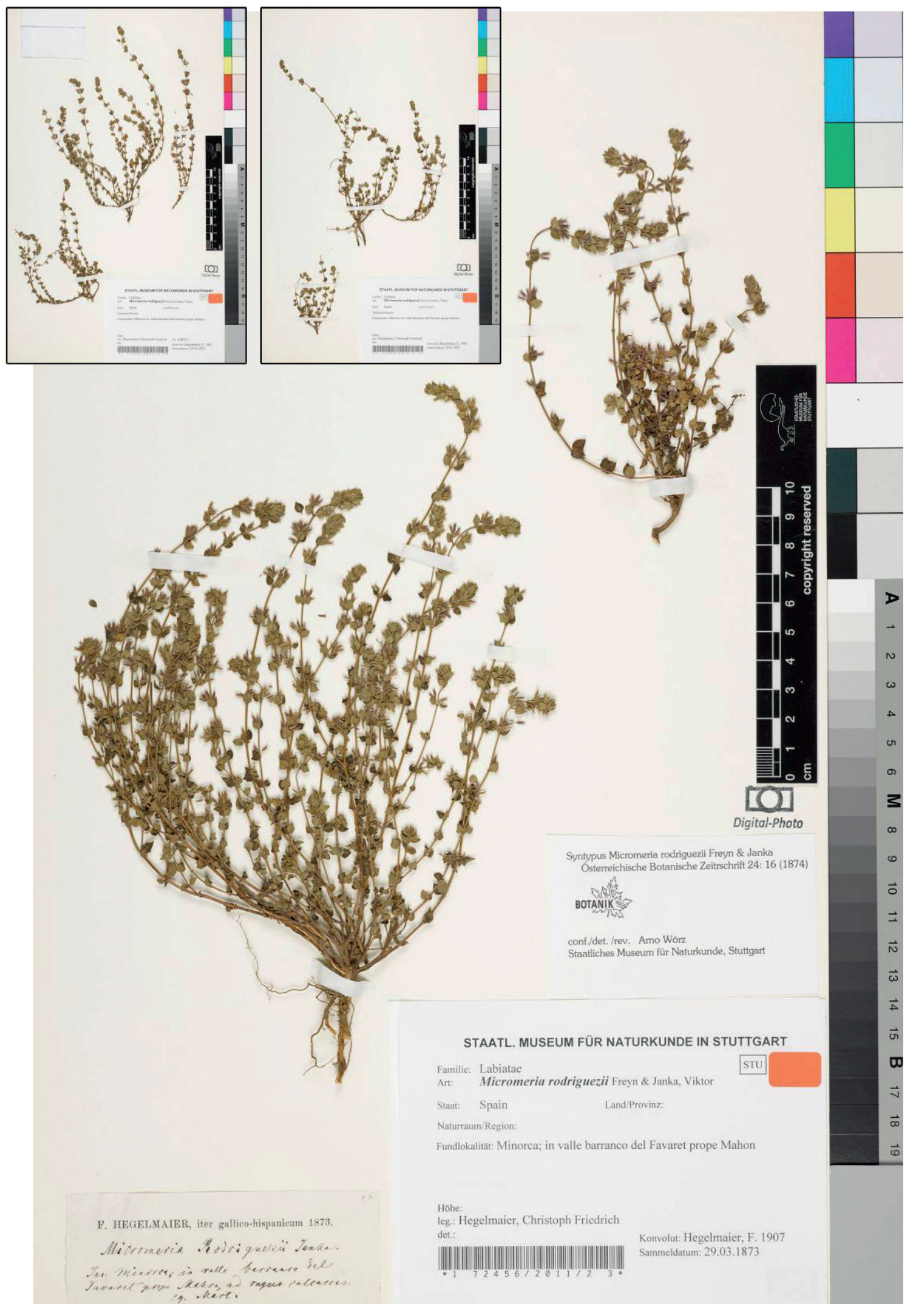

Figura 2. Lectótipo de Micromeria rodriguezii Freyn \& Janka, designado por Bräuchler et al. (2008), STU [imagen grande: código de barras STU 1 72456/2011/2 3 (hoja 2); imágenes pequeñas: STU 1 72456/2011/1 3 (hoja 1) y STU 1 72456/2011/1 3 (hoja 3)]. Imágenes por cortesía de herbario STU, reproducidas con permiso. 


\section{MATERIAL Y MÉTODOS}

Para el estudio morfológico se recopilaron datos a partir de la bibliografía consultada, así como de los ejemplares recolectados en campo y especímenes de herbario (ver apartado Specimina visa selecta). Los acrónimos de los herbarios mencionados en el presente trabajo aparecen de acuerdo a lo indicado por Thiers (2019).

\section{RESULTADOS Y DISCUSIÓN}

Micromeria rodriguezii Freyn \& Janka, Osterr. Bot. Z. 24: 16 (1874).

España, Castellón: Santa Magdalena de Polpís, camí del Saltet, pr. Mas de Xarpa, 31TBE732720 (ETRS89), $120 \mathrm{~m}$, depressió de desguàs de l'aigua de 1'AP-7, pastures d'anuals sobre gleres calcàries, R. Senar, 26.VIII.2018 (VAL 241026) (Figs. 3 y 4); ibídem, camí de la autopista (margen derecha en sentido Tarragona), 31TB732719, $124 \mathrm{~m}$, B. Plana, P.P. Ferrer-Gallego \& E. Laguna [recolección realizada durante la XIV Semana de la Biodiversidad], 6.V.2019 (VAL 242218).

Pequeño caméfito de hasta $8-22 \mathrm{~cm}$ de altura, ramificado en la base; ramillas largas y finas que crecen desde la base, 0,7-1 $\mathrm{mm}$ de grosor, erectas $\mathrm{o}$ arqueado-erectas, pubescentes, con indumento eglandular de pelos retrorsos, de 0,1-0,3(0,4) $\mathrm{mm}$, más o menos densamente dispuestos. Hojas $(1,2) 2-$ $3(3,5) \times 1-2 \mathrm{~mm}$, pecioluladas $(\mathrm{ca} .0,5 \mathrm{~mm})$, ovadas, agudas, redondeadas en la base, en ocasiones cordadas, las superiores de color verde en el envés, más estrechas, y las basales generalmente de color púrpura, más anchas; márgenes engrosados, en las hojas basales en ocasiones levemente revolutos; dispersamente pelosas, con pelos eglandulares antrorsos de color blanco-cinéreo, pelos pequeños ( $c a .0,1$ $\mathrm{mm}$ ) y abundantes mezclados con otros más escasos y de mayor tamaño (de hasta $0,5 \mathrm{~mm}$ ), estos últimos dispersos por el haz, y algo más concentrados en el nervio medio en el envés, con presencia de glándulas sésiles en el haz y envés. Verticilastros formados por dos fascículos pedunculados, con pedúnculo de hasta 2,5 mm, con 2-5(8) flores por fascículo; pedicelos de hasta $1,8 \mathrm{~mm}$; bractéolas de ovadas a ovado-lineares, de hasta $1 \times 0,5 \mathrm{~mm}$, al menos una por pedicelo, que crecen en la base de éstos. Cálices 3(3,3) mm de longitud, con dientes de hasta $1 \mathrm{~mm}$ de longitud, tubo fino en la floración y algo engrosado cuando contiene núculas, pelosos, con abundantes pelos eglandulares en tubo y dientes, de hasta $0,4 \mathrm{~mm}$, antrorsos, rígidos, blanco-cinéreos, con mayor densidad en las costillas, presencia de glándulas sésiles. Corolas $c a$. $5,5 \mathrm{~mm}$, rosadas, abiertas o con flores cleistógamas (al menos durante otoño e invierno). Núculas ca. 0,9 $\times 0,5 \mathrm{~mm}$, elipsoidales, marrones.

El material de Castellón (Figs. 3 y 4) es muy próximo desde el punto de vista morfológico al herborizado por Friedrich Hegelmaier, el cual fue empleado por Freyn y Janka para la descripción de $M$. rodriguezii (véase JE 00007503, STU 1 72456/2011/1 3, STU 1 72456/2011/2 3 y STU 1 $72456 / 2011 / 3$ 3, véase Fig. 2), así como otro conservado en varios herbarios, procedente de Menorca (Ferreries, barranco de Algendar: VAL 225004, MA-01-00874721. Mercadal, Monte Toro, Sant Nicolau: VAL 225354, MA-01-00876636. Trepucó, BC 49866), o Mallorca (Route de Valdemosa: MA 104550-1-2, BC-Sennen 836596, W 19840000218. De Palma a Valdemosa: BC-Sennen 836599. Torre d'en Pau: G00087103. Costes de Xorrigo, L. Sáez, herb. pers.) el cual determinamos como perteneciente a $M$. rodriguezii, aunque suele aparecer en la mayoría de los casos identificado en las etiquetas de herbario como M. microphylla (véase además el apartado Specimina visa selecta).

Bolòs \& Vigo (1996: 301) separan M. microphylla s. str. de M. rodriguezii (tratado por estos autores como Satureja microphylla subsp. rodriguezii) por tener la primeras hojas no cordadas y pedicelos no geniculados. Por otra parte, Bolòs \& Vigo (1996) consideran $M$. microphylla una planta que no se encontraría presente en las Islas Baleares. Estos autores diferencian el taxón que aquí nos ocupa de M. filiformis [tratado por estos autores como Satureja microphylla subsp. filiformis (Aiton) O. Bolòs \& Vigo] por ser una planta algo más pubescente, de tallos algo más robustos y en general erectos o suberectos, hojas poco purpureas en el envés, con 2-8 flores en la axila de las hojas, y flores rosadas, mientras que $M$. filiformis $s$. str. resultaría ser algo más glabra, de tallos más gráciles, con hojas generalmente de color púrpura en el envés, 1-2 flores en las axilas de las hojas y flores blancas o rosadas. Por otra parte, Pignatti (2018) atribuye a M. microhylla indumento de pelos patentes de $0,5 \mathrm{~mm}$ de longitud, hojas inferiores de lanceoladas a ovadas y pedicelos casi erectos, siendo geniculados en $M$. 


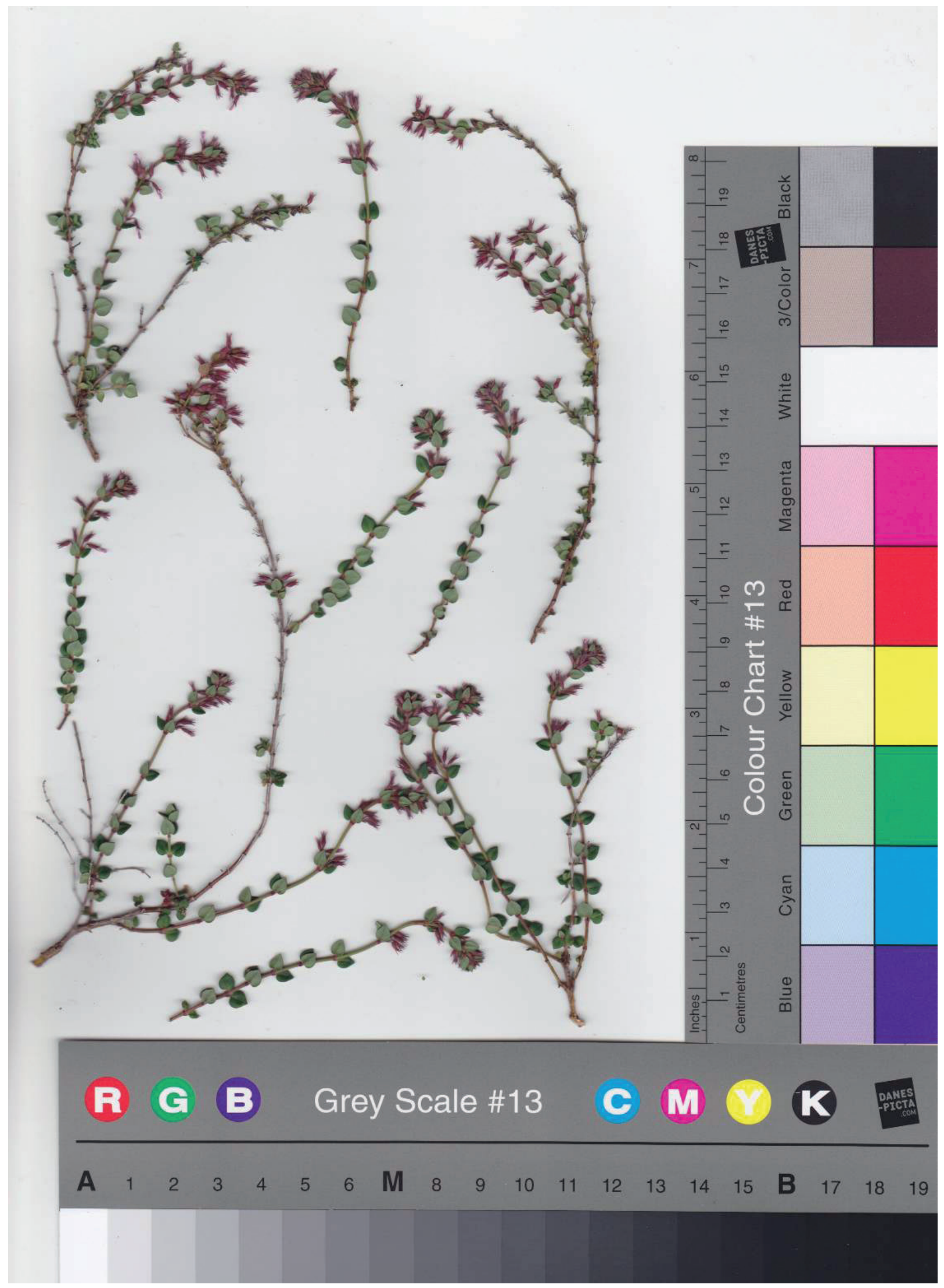

Figura 3. Pliego de herbario de Micromeria rodriguezii con material recolectado en Castellón, Santa Magdalena de Polpís (VAL 241026). 

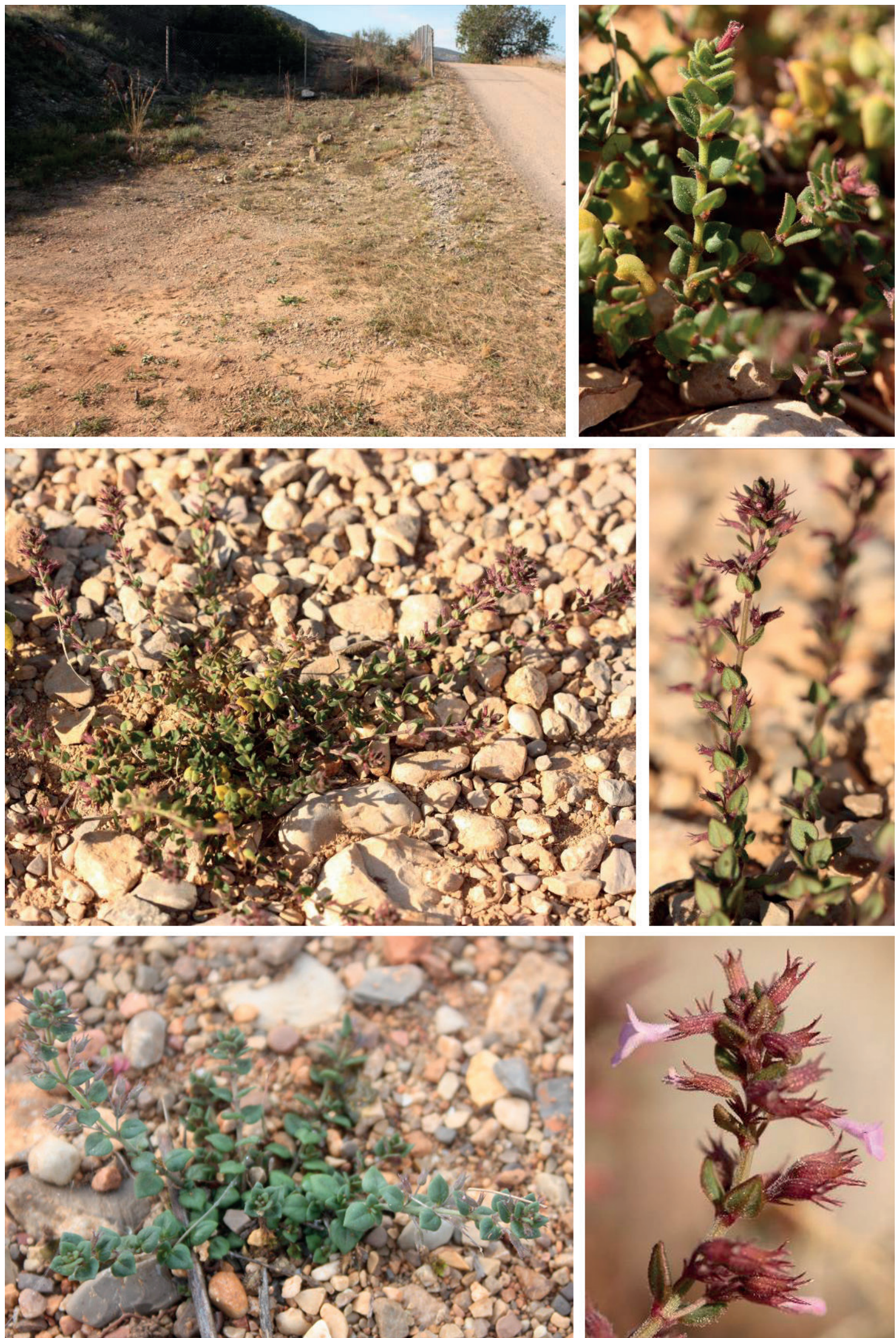

Figura 4. Hábitat y detalles de las hojas e inflorescencias y porte de las plantas de Micromeria rodriguezii presente en la población castellonense de Santa Magdalena de Polpís. 
rodriguezii; además diferencia $M$. rodriguezii de M. cordata por presentar, esa última, hojas ovadas a ovato-cordadas y corola banca, siendo rosa o rosada en $M$. rodriguezii.

Además de lo indicado por Bolòs \& Vigo (1996) y Bolòs et al. (2005) para la diferenciación entre estos táxones, nuestras observaciones indican que M. filiformis es una especie glabrescente, con pelos eglandulares retrorsos en los tallos, de 0,1-0,2(0,3) $\mathrm{mm}$, hojas cordadas, purpúreo-violáceas en el envés, con pelos erectos o antrorsos de 0,1-0,2(0,3) $\mathrm{mm}$; flores en grupos de 1-2; cáliz con pelos eglandulares escasos en el tubo y cortos (de hasta 0,2 $\mathrm{mm}$ ), más densos en los dientes; corola blanca o de un rosa pálido; mientras que $M$. rodriguezii es una planta más densamente pelosa, con pelos eglandulares retrorsos en tallo de $0,1-0,3(0,4) \mathrm{mm}$, hojas ovadas, redondeadas en la base, en ocasiones cordadas (esto se ha observado tanto en las muestras procedentes de Castellón como en diversos materiales baleáricos depositados en $\mathrm{BC}$ ), por lo general verdes en el envés, con pelos eglandulares antrorsos de hasta $0,5 \mathrm{~mm}$, flores en grupos de 2-5(8); cáliz de 3,5-4,5 mm, con abundantes pelos eglandulares en tubo y en los dientes (de hasta $0,6 \mathrm{~mm}$ ), con presencia de glándulas sésiles; corola rosada.

En lo relativo al indumento, éste es un carácter bastante variable; Mus \& Rosselló (1987) consideran que las plantas italianas pertenecientes al complejo de M. microphylla son ligeramente más pubescentes que las procedentes de las Islas Baleares. Sin embargo, existen muestras procedentes de Malta (de donde se describió M. microphylla) que son bastante más pubescentes que las baleáricas y que el material de Castellón. En este sentido, autores como Greuter et al. (1986) y Bolòs \& Vigo (1996) aceptan la planta descrita por Freyn y Janka como representante del grupo de M. microphylla en las Islas Baleares.

Ecología y corología: en las Islas Baleares, esta especie habita en colinas pedregosas, lugares yermos, roquedos, bordes de caminos, generalmente sobre calizas y en ocasiones en margas y lugares algo arenosos. La población de Castellón crece en suelo calizo, pedregoso y compactado. La vegetación general de la zona corresponde a la propia de un coscojal termófilo, pero ambas poblaciones aparecen en ambiente algo antropizado, compartiendo hábitat junto con otros caméfitos y especies de ciclo anual propias de pastizales cársticos ruderalizados, como: Ajuga iva (L.) Schreb., A. chamaepitys
(L.) Schreb., Asphodelus fistulosus L., Bituminaria bituminosa (L.) C. H. Stirt., Eragrostis barrelieri Daveau, Fumana ericifolia Wallr., F. thymifolia Spach, Hippocrepis ciliata Willd., Hyparrhenia hirta Stapf, Leontodon longirostris (Finch \& P. D. Sell) Talavera, Linum strictum L., Lobularia maritima (L.) Desv., Lysimachia arvensis (L.) U. Manns \& Anderb., Minuartia hybrida (L.) Schischk., Piptatherum coerulescens P. Beauv., Phagnalon rupestre (L.) DC., P. saxatile (L.) Cass., Plantago afra L., P. lagopus L., Sanguisorba verrucosa (Link ex G. Don) Ces., Scorpiurus sulcatus L., Sherardia arvensis L., Stachys ocymastrum Briq., Trigonella monspeliaca L. (Fig. 4). En ambos casos se sitúan en bordes de pistas o caminos, ocupando claros donde se disponen los terófitos entre las especies multianuales. Estos dos núcleos se sitúan a ambos lados de la autopista AP7 junto a un paso de agua que transcurre bajo esa vía de comunicación, por lo que es muy probable que en el pasado formaran una única población ahora fragmentada, o que uno de ellos derivara de la expansión del otro al arrastrar el agua sus semillas a través del paso de agua.

No se descarta que en un futuro puedan aparecer más ejemplares en otros medios forestales, situados a poca distancia de estos dos núcleos poblacionales, sobre todo en los claros del matorral y pinar situados en las laderas pedregosas de las sierras del Parque Natural de la Serra d'Irta.

Florece desde marzo a octubre y fructifica desde mayo a noviembre. Su principal floración ocurre en la primavera y el verano, aunque fuera de estas estaciones, si la humedad edáfica es importante, se prolonga su floración produciendo flores cleistógamas (Fig. 4), probablemente como respuesta a las bajas temperaturas.

Estatus de conservación: este hallazgo representa la primera referencia de esta especie en la Península Ibérica, hasta ahora considerada endemismo exclusivo de las Islas Baleares. El grado de aislamiento respecto de las poblaciones baleáricas confiere a la población de Castellón una elevada singularidad biogeográfica. Consideramos importante señalar que, a pesar de la localización de esta población cercana a una vía de comunicación, no existen evidencias sólidas que ésta haya sido introducida. No obstante, la localización de esta población en un ambiente antropizado y próximo a un camino con cierta densidad de tránsito de vehículos, puede llegar a sugerir que se trate de una colonización 
reciente. Un estudio más intenso del entorno natural próximo podrá aportar más información sobre el origen de esta población.

Por otra parte, el bajo número de individuos localizados (25-30 ejemplares adultos en el núcleo del camí del Saltet y 170-190 individuos adultos en el núcleo del camino de la autopista), junto con su reducidísima área de ocupación, menor de 60 $\mathrm{m}^{2}$ entre los dos núcleos (aprox. $20 \mathrm{~m}^{2}$ en el núcleo del camí del Saltet y aprox. $40 \mathrm{~m}^{2}$ en el núcleo del camino de la autopista), y el fuerte riesgo de afecciones negativas al situarse ambos núcleos en el margen de un camino, y una de ellas (camí de la autopista) junto a campos de cultivo, nos sugiere proponer su inclusión en el Catálogo Valenciano de Especies de Flora Amenazadas en la categoría de "En peligro de extinción" (CGV, 2009, 2013).

Como medidas de conservación para esta especie, por el momento se ha recolectado material vegetal de reproducción, y así se conservan semillas en el Banco de Germoplasma de la Flora Silvestre Valenciana en el CIEF (Centro para la Investigación y Experimentación Forestal de la Generalitat Valenciana) y se mantienen ejemplares en cultivo dentro de una colección de planta viva mantenida en condiciones ex situ en las instalaciones del CIEF.

\section{Specimina visa selecta}

\section{Micromeria cordata}

Italia: Cerdeña, s.d., Moris s.n., BOLO (lectótipo) (Fig 1).

\section{Micromeria filiformis}

España: Islas Baleares, s.d., Jacquin s.n., BM (Herbarium Banks) (lectótipo).

\section{Micromeria microphylla}

Italia: Sicilia, Siracusa, Belvedere, 6.IV.1960, I. Segelberg s.n. (MA 523472). Lampedusa, Cala Uccello, 1905, U. Martelli s.n. (MA 104567). Herbarium Vaillant (P04049884).

\section{Micromeria rodriguezii}

España, Islas Baleares, Menorca: Mahón, 1898, J.J. Rodriguez s.n. (MA 104543). Fuente de Marlborough, cala de San Esteve, 30.IV.2013, R. Morales 2560 et al. (MA 876146). Mercadal, Monte Toro, Sant Nicolau, 2.V.2013, P. Barberá 547 et al. (MA 876636). Ciudadela, Naveta des Tudons,
P. Barberá 575 et al. (MA 876585). Menorca, Ferreries, 29.IV.2013, C. Aedo 20096 et al. (MA 874721). Menorca, s.d., Alabau s.n. (MA 104539). Mahón, 11.IX.1913, Font Quer 920 (MA 486766). San Clemente, 24.XII.1913, Font Quer 917 (MA 104548). San Vilá, c. Mahón, 6.III.1914, Font Quer 919 (MA 104549). Mallorca: Palma, Castillo de Bellver, IX.1933, C. Pau s.n. (MA 104547). Palma de Mallorca, parque del Castillo de Bellver, 11.X.1989, R. Morales 586, Erben \& Rosselló (MA 486678). Palma, chemins et collines autor de Palma, 11.VII.1914, F. Bianor s.n. [exsiccata Plantes d'Espagne - F. Sennen N. $\left.{ }^{\circ} 2037\right]$ (MA 104542-12). Pont d'Inca, Torrent Gros, 2.V.1949, P. Ferrer 166 (MA 341963). Palma, 1907, Mas Guindal s.n. (MA 104545). Collines de Palma, 26.XII.1912, F. Bianor s.n. [exsiccata Plantes d'Espagne - F. Sennen N. ${ }^{\circ}$ 1422] (MA 104544-1-2). Palma, 1899, Gandoger s.n. (MA 104546). Palma de Mallorca, 20.III.1918, Font Quer s.n. (MA 104540). Palma, 12.IV.1933, F. Sennen s.n. (MA 104541). Mallorca, Alcudia, 20.VI.1980, S. Castroviejo $1965 \& R$. Morales (MA 486765). Palma, 4.IX.194?, P. Ferrer s.n. (MA 104537). Can Pastilla, 20.IV.1947, P. Ferrer s.n. (MA 104408). Mallorca, Sa Ràpita, playa de Ses Covetes, 3.VI.1998, C. Navarro 2125 \& al. (MA 618666). Palma, Puig Rodò, Sa Vileta, Son Quint, 3.I.1999, L. Sáez 5177 (MA 623478). Almedrá a Tossal Verds, 4.VI.1998, C. Aedo 4579 \& al. (MA 619699). Castell de Santueri, Felanitx, 24.IV.2000, L. Sáez 5346 (MA 648645) sub Micromeria rodriguezii f. major Sennen. España: Islas Baleáres, Route de Valdemora, à $6 \mathrm{~km}$. de Palma, 13.VII.1914, F. Bianor [exsiccata Plantes d'Espagne - F. Sennen N. ${ }^{\circ}$ 2036] (MA 104550-1-2) (isolectótipo [dos hojas]) sub Micromeria $\times$ knochei Sennen \& Bianor. España: Islas Baleáres, Collines près de Palma, V.1913, F. Bianor [exsiccata Plantes d'Espagne - F. Sennen N. ${ }^{\circ}$ 2039] (MA 104469-1-2) (isolectótipo) (BC-Sennen 836597) (lectótipo).

\section{AGRADECIMIENTOS}

Los autores agradecen a U. Mossetti (Botanic Garden and Herbarium of the University of Bolonia, Italia, BOLO), A. Rosenbauer y M. Thiv (Staatliches Museum für Naturkunde Stuttgart, STU) toda la ayuda prestada para el estudio de los materiales de herbario solicitados. L. Medina y C. Baranda prestaron toda la ayuda en el estudio de los pliegos de herbario conservados en MA durante la estancia de uno de los autores (PPFG) en el Real Jardín Botánico de Madrid. 


\section{REFERENCIAS BIBLIOGRÁFICAS}

Bentham, G. 1829. Micromeria Benth., gen. nov. In: Lindley, J. (Ed.), Edward's Botanical Register 15. London: 1282. Consultado en octubre de 2019, en http://www.biodiversitylibrary.org/item/9051\#page/193/mode/1up

Bolòs, O. de \& Vigo, J. 1996. Flora dels Països Catalans 3. Barcino, Barcelona.

Bolòs, O. de, Vigo, J., Masalles, R. M. \& Ninot, J. M. 2005. Flora manual dels Països Catalans (3a ed.). Pòrtic, Barcelona.

Bräuchler, C., Meimberg, H., Abele, T. \& Heubl, G. 2005. Polyphyly of the genus Micromeria Benth. (Lamiaceae) evidence from cpDNA sequence data. Taxon 54: 639-650. https://doi.org/10.2307/25065421

Bräuchler, C., Ryding, O. \& Heubl, G. 2008. The genus Micromeria (Lamiaceae), a synoptical update. Willdenowia 38: 363-410. https://doi.org/10.3372/wi.38.38202

CGV [Consell de la Generalitat Valenciana] 2009. Decreto 70/2009, de 22 de mayo, del Consell, por el que se crea y regula el Catálogo Valenciano de Especies de Flora Amenazadas y se regulan medidas adicionales de conservación. Diari Oficial de la Generalitat Valenciana 6021: 20143 20162.

CGV [Consell de la Generalitat Valenciana] 2013. Orden 6/2013, de 25 de marzo, de la Conselleria de Infraestructuras, Territorio y Medio Ambiente, por la que se modifican los listados valencianos de especies protegidas de flora y fauna. Diari Oficial de la Generalitat Valenciana 6996: 8682-8690.

Chater, A. O. 1971. Labiatae. (193) Micromeria Bentham. In: Heywood, V. H. (Ed.), Flora Europaea, Notulae Systematicae ad Floram Europaeam spectantes No. 11. Botanical Journal of the Linnean Society 64: 381. https://doi. org/10.1111/j.1095-8339.1971.tb02152.x

Chater, A. O. \& Guinea, E. 1972. Micromeria Bentham. In: Tutin, T. G., Heywood, V. H., Burges, N. A., Moore, D. M., Valentine, D. H., Walters, S. M. \& Webb, D. A. (Eds.), Flora europaea 3. Cambridge University Press, Cambridge: 167-170.

Fraga-Arguimbau, P. 2015. Notes i contribucions al coneixement de la flora de Menorca (XI). L'herbari d'Agustí Landino Flores (1875-1950), una contribució inèdita a la flora de Menorca. Bolletí de la Societat d'Historia Natural de les Balears 58: 45-90.

Fraga-Arguimbau, P., Estaún, I., Comas, M., \& Cardona, E. 2014. Plantes de Menorca. Consell Insular de Menorca, Institut Menorquí d'Estudis, Maó.

Fraga-Arguimbau, P., Mascaró, C., Carreras, D. et al. 2004a. Catàleg de la flora vascular de Menorca. Institut Menorquí d'Estudis, Consell Insular de Menorca, Maó.

Fraga-Arguimbau, P., Truyol, M., Mascaró, C., Carreras, D., Gracia, O., Pons, M. \& Seoane, M. 2004b. La vegetació del Migjorn de Menorca: un passeig descriptiu. In: Fornós, J. J., Obrador, A. \& Rosselló, V. M. (Eds.), Història natural del Migjorn de Menorca: el medi fisic i l'influx humà (Monografies de la Societat d'Història Natural de les Balears, 11). Institut Menorquí d'Estudis, Fundació Sa Nostra, Palma de Mallorca: 53-72.

Freyn, J. \& Janka, V. de 1874. Micromeria (Satureja) Rodriguezii. Nov. spec. e sect. Piperella DC. prodr. Oesterreichische Botanische Zeitschrift 24: 16-18. https://doi. org/10.1007/BF01963496

Gil, L. \& Llorens, L. 2004. Análisis biogeográfico de la flora de Formentera (Islas Baleares, España). Lazaroa 25: 169-178.
Govaerts, R. 1999. World checklist of seed plants 3. MIM, Deurne.

Greuter, W., Burdet, H. M. \& Long, G. (Eds.) 1986. MedChecklist. A critical inventory of vascular plants of the circum-mediterranean countries 3. Dicotyledones (Convolvulaceae - Labiatae). Conservatoire et Jardin Botanique, Genève.

Harley, R. M., Atkins, S., Budantsev, A. et al. 2004. Labiatae. In: Kadereit, J. W. (Ed.), The families and genera of flowering plants 7 . Springer, Berlin \& Heidelberg: 167-275. https://doi.org/10.1007/978-3-642-18617-2 11

Morales, R. 1991. El género Micromeria Bentham (Labiatae) en la Península Ibérica e Islas Baleares. Anales del Jardín Botánico de Madrid 48: 131-156.

Morales, R. 1993. Sinopsis y distribución del género Micromeria. Botanica Complutensis 18: 157-168.

Morales, R. 2010. Micromeria Benth. In: Morales, R., Quintanar, A., Cabezas, F., Pujadas, A. J. \& Cirujano, S. (Eds.), Flora iberica 12. Real Jardín Botánico, CSIC, Madrid: 423-430.

Mus, M. \& Rosselló, J. A. 1987. Micromeria microphylla (D’Urv.) Benth. en España. Anales del Jardín Botánico de Madrid 43: 459-460.

Pérez de Paz, P. 1978. Revisión del género Micromeria Bentham (Lamiaceae-Stachyoideae) en la región Macaronésica (Monografías del Instituto de Estudios Canarios, 16). Instituto de Estudios Canarios, La Laguna.

Pignatti, S. 2018. Micromeria Bentham. In: Flora d'Italia 3. Edagricole, Milano: 236-239.

Puppo, P., Curto, M., Gusmão-Guedes, J., Cochofel, J., Pérez de Paz, P. L., Bräuchler, C. \& Meimberg, H. 2015. Molecular phylogenetics of Micromeria (Lamiaceae) in the Canary Islands and Madeira, diversification and inter-island colonization patterns inferred from nuclear genes. Molecular Phylogenetics and Evolution 89: 160-170. https://doi. org/10.1016/j.ympev.2015.04.017

Puppo, P., Curto, M., Velo-Antón, G., Pérez de Paz, P. L. \& Meimberg, H. 2014. The influence of geological history on diversification in insular species: genetic and morphological patterns of Micromeria Benth. (Lamiaceae) in Tenerife (Canary archipelago). Journal of Biogeography 41: 18711882. https://doi.org/10.1111/jbi.12354

Puppo, P. \& Meimberg, H. 2015. New species and new combinations in Micromeria (Lamiaceae) from the Canary Islands and Madeira. Phytotaxa 230: 1-21. https://doi. org/10.11646/phytotaxa.230.1.1

Rivas-Martínez, S., Costa, M. \& Loidi, J. 1992. La vegetación de las islas de Ibiza y Formentera. Itinera Geobotanica 6: 99-236.

Rosselló, J. A. \& Sáez, L. 2000. Index Balearicum: An annotated check-list of the vascular plants described from the Balearic Islands. Collectanea Botanica 25: 3-203. https:// doi.org/10.3989/collectbot.2000.v25.42

Rosselló, J. A. \& Sáez, L. 2017. Index Balearicum (III). An annotated check-list of the vascular plants described from the Balearic Islands. Additions (2008-2016) and corrections. Orsis 31: 65-78.

Sennen, F. 1917. Flore de Catalogne. Additions et commentaires. Treballs de la Institució Catalana d'Història Natural 3: 55-266.

Thiers, B. 2019. Index Herbariorum: A global directory of public herbaria and associated staff. New York Botanical Garden's Virtual Herbarium, New York. Consultado en octubre de 2019, en http://sweetgum.nybg.org/ih/ 